\title{
Microfluidic discharge-based optical sources for detection of biochemicals
}

\author{
Bhaskar Mitra, Chester G. Wilson, Long Que, P. Selvaganapathy and Yogesh B. Gianchandani*
}

Received 19th July 2005, Accepted 16th November 2005

First published as an Advance Article on the web 2nd December 2005

DOI: 10.1039/b510332k

This paper reports a discharge-based optical source for fluorescence of biochemicals in microfluidic systems. Its efficacy is demonstrated using a stacked microchip that integrates a microfluidic wavelength-tunable optical source, a biochemical sample reservoir and optical filters. It is shown to excite fluorescence in L-tryptophan and DNA samples labeled by SYBR green dye. The discharge is struck in ambient air, between a metal anode and a cathode cavity that is filled with an aqueous solution, which is doped with a metal salt selected for its emission characteristics. The characteristic line spectra, which arise from energetic transitions of the metal ions that are sputtered into the glow region of the discharge, are optically filtered and guided to the biochemical sample that resides in a separate on-chip reservoir. For DNA fluorescence, a barium chloride solution is used to emit light at 454 and $493 \mathrm{~nm}$. For tryptophan fluorescence, the cathode contains lead (II) nitrate solution to provide a $280 \mathrm{~nm}$ emission. The resulting fluorescence from the DNA and tryptophan samples is compared to reference data. This technique can also be used to excite other fluorophores by using appropriately doped liquid cathodes having the desired emission characteristics.

\section{Introduction}

Fluorescence detection is a widely used technique in medical diagnostics and biochemical analysis. Many "fluorescent molecules" emit light with characteristic wavelengths when illuminated by certain excitation wavelengths, which are shorter (and hence more energetic). In one diagnostic approach, a fluorescent dye is used to chemically label the analyte of interest. For DNA detection, dyes which intercalate into the double-helix provide very high sensitivity and make it possible to detect even attomoles of DNA base-pairs. ${ }^{1}$ A major reason for the high sensitivity is quantum efficiency, which is the ratio of the number of photons emitted to those absorbed in the excitation wavelengths. For example, SYBR Green I gel stain is a cyanine dye that has a quantum efficiency of $0.8 .^{2}$ When bound to dsDNA it is most efficiently excited by radiation over 491-503 $\mathrm{nm}$, and has a broad emission spectrum over $510-600 \mathrm{~nm}$, with a peak at $522 \mathrm{~nm}$. In addition to DNA, fluorescence detection using dyes can be used to ascertain the concentration of many other compounds (like glucose, ATP, RNA, proteins, oxygen, carbon dioxide) in the cellular microenvironment.

While dyes offer many attractive features, their use is not always favored or even possible. Most dyes suffer photobleaching ${ }^{3}$ after many exposures and can be toxic to the cell. Hence, in many of these cases where biological activity still needs to be monitored the intrinsic fluorescence of biological molecules is used. For example NADH, which is a product in the cell metabolic cycle, can be used to monitor the metabolism of a cell. ${ }^{4}$ In addition, many proteins are fluorescent even without the presence of a dye, and changes in this intrinsic or

Department of Electrical Engineering and Computer Science, University of Michigan, Ann Arbor, MI 48108-2122, USA.

E-mail: yogesh@umich.edu; Fax: +1 763-9324; Tel: +1 734-615-6407 direct fluorescence can be indicative of structural transformations. 5 The intrinsic fluorescence of such proteins and peptides is due to the presence of tryptophan, tyrosine or phenylalanine, which are fluorescent amino acids. In contrast to the excitation and emission wavelengths for the SYBR green dye, which are in the visible portion of the spectrum, these three have absorption peaks over 250-290 $\mathrm{nm}$ and emission peaks over 280-350 nm, all in the deep ultra-violet (UV) region. The intrinsic fluorescence of tryptophan, in particular, has been widely used in studying proteins, due to its strong dependence on protein structure and dynamics. However, the quantum efficiencies of these amino acids are relatively low. For example, tryptophan, which tends to dominate in fluorescence over the other two, has a quantum efficiency of only 0.19 when dissolved in water as a free amino acid. ${ }^{5}$ So to observe direct fluorescence, not only do the sources need to emit in deep-UV region of the spectrum but also provide sufficient optical power and have a small footprint to be easily integratable with microsystems.

In the recent past tremendous progress has been made in microfluidics and micro total-analysis systems. These systems, however, still rely on relatively large external light sources for fluorescence detection, due to lack of good miniature light sources. Alternatives to optical detection, based on change in ion-concentration, impedance or mechanical properties have been implemented because of the problems with optical sources. Although these are very good for certain applications like monitoring $\mathrm{pH}$, they lack the specificity of fluorescence techniques, do not provide spatial information and are restricted to a few chemical species only.

With respect to fluorescence detectors, the efforts have focused on solid-state sources such as light-emitting diodes (LEDs) and lasers (VCSELs). ${ }^{7-12}$ However these do not have strong emission in the blue and UV region where most of the 
fluorescent chemicals have their excitation spectrum. There has been some success in using AlGaN heterojunction LEDs for deep UV light. ${ }^{11,12}$ Although they emit in UV region, the emission lies in a very narrow region of the spectrum and it is difficult to tune its wavelength to different fluorescent molecules. In addition, these can only be made on sapphire substrates and require sophisticated fabrication processes and equipment, and hence, are not disposable. Further, integrating them with low-cost microfluidic systems can be a significant challenge.

In the context of this paper, microdischarges are minute electrical discharges, typically generated by electrical breakdown of air across a small gap between two metal electrodes. While this term has also been used to describe small plasmas, in this work the discharges are like arcs. Although microdischarges do share some characteristics with larger scale discharges, they also have some distinguishing characteristics in the underlying physics and properties. ${ }^{13}$ One of the appealing features is that they have promising emission characteristics in the UV region, ${ }^{13-19}$ and can easily and inexpensively be integrated with microfluidic systems. It has previously been reported that spectroscopy on microdischarges can be used to sense trace amounts of inorganic impurities in aqueous samples. ${ }^{14-16}$ In such devices, the microdischarge sputters the ions from the surface of the liquid cathode into its glow region. Atomic transitions from excited ions and atoms in the microdischarge lead to emission of characteristic spectra. In this work, the microdischarge is used as a tunable optical source by using a liquid solution of predetermined composition (instead of an unknown sample), targeted to generate specific wavelengths. The microdischarge is driven by a custom built circuit which is powered by a $3 \mathrm{~V}$ battery, and hence suitable for portable applications. The device operates in ambient atmosphere and does not use inert gases. The design of the device and circuit, and the fabrication process are described in section II. The experiments used to validate the circuit and the device, including the performance evaluation of the device in two application scenarios are presented in section III.

\section{Device structure and fabrication}

A schematic of the microdischarge device is shown in Fig. 1. The electrical microdischarge is ignited across an air gap between a metal anode and a liquid reservoir containing a saturated salt solution, which serves as the cathode. The metal ions in the cathode are sputtered into the glow region of the discharge and emit a characteristic glow. ${ }^{16}$ Fluid heating and vaporization are minimal under the use conditions, ${ }^{16,20}$ so thermal isolation is not required. The saturated solution is carefully chosen to cause emission in the required wavelength range. Unwanted wavelengths are rejected by an optical filter that separates the microdischarge from the fluorescent sample. A dichroic color filter is chosen as the integrated band pass filter due to its low sensitivity to the light incident angle and its superior lifetime and reliability over other types of filters (e.g., interference filter). The sample is located in a reservoir or channel as part of a micro-total analysis system. The filtered emission from the discharge pulse follows optical path I

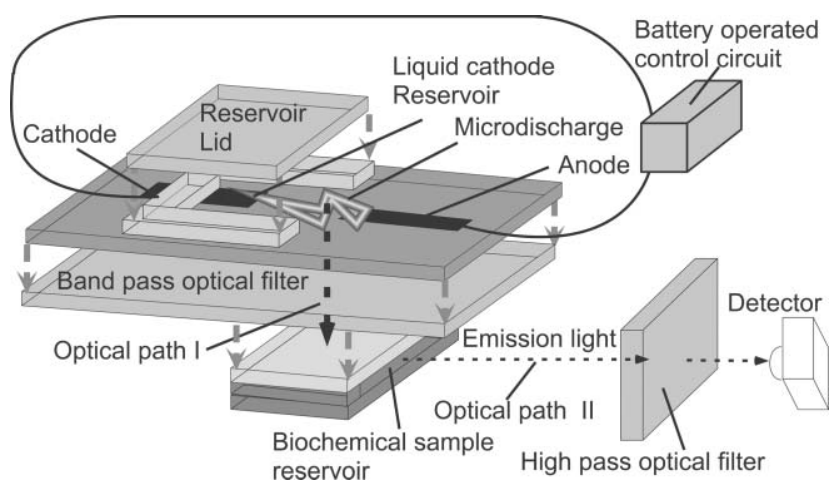

Fig. 1 Exploded schematic of the liquid electrode discharge spectral emission chip (LEdSpEC), which is a stacked microchip, and the associated control circuit and detector arrangement. Short arrows indicate the stacking order for the microchip whereas long arrows indicate the two orthogonal optical paths, as noted. The chip is held vertically during operation.

(Fig. 1), and stimulates fluorescence in the sample reservoir, which is detected along optical path II. These paths are orthogonal to minimize the signal feed-through from the arc to the detector, which is an Ocean Optics USB $2000^{(i x}$ spectrometer that connects to the USB port of a computer.

The stacked device was fabricated by bonding the individual glass layers using UV-curable epoxy (Lens Bond, type SK-9 optical cement from Summers optical, PA, USA) which has the same index of refraction as glass. All the glass layers were $506 \mu \mathrm{m}$ thick, \#7740 Pyrex $^{(i i}$ glass which has transmittance $>80 \%$ for $300-700 \mathrm{~nm}$ wavelengths. A $4 \mathrm{~mm}$ wide strip of MetGlas ${ }^{\circledR}$ foil (Magnetic Alloy 2826 MB from Metglas Inc.) was used as the anode for its superior wear-resistant properties. The cathode cavity was $4 \mathrm{~mm} \times 5 \mathrm{~mm} \times 0.5 \mathrm{~mm}$ in size, and the discharge gap was $0.4 \mathrm{~mm}$. The cathode contact was also a MetGlas foil placed $3 \mathrm{~mm}$ deep inside the cathode cavity. The optical filter was a commercial dichroic filter made on a $2.5 \mathrm{~mm}$ thick quartz substrate. The biochemical reservoir was $4 \mathrm{~mm} \times 2.5 \mathrm{~mm} \times 0.5 \mathrm{~mm}$, and was bonded to the filter at the other end. The biochemical reservoir was aligned with its center in line with the discharge gap to maximize the amount of light coupling from the discharge to the biochemical reservoir. The biochemical reservoir can be much smaller and can be a part of an overall microfluidic system. Microfluidic and other kinds of lenses can also be incorporated into the light path to focus the light from the microdischarge to a point. The cathode cavity can also be part of a more complex microfluidic system having many reservoirs filled with different chemicals, connected to the cathode cavity with channels and incorporating pumps and valves to control the flow. Such a system allows real time tuning of the light output during operation. Although non-lithographic techniques have been used in its current manifestation, the device can also be made using thin film electrodes and lithographic techniques (as demonstrated in some of our previous work, notably ref. 16).

A photograph of the final fabricated device is shown in Fig. 2. The overall footprint of the device is approximately $10 \mathrm{~mm} \times 20 \mathrm{~mm}$, with the reservoirs covering half the area. The assembly is held horizontally during operation, with the detector placed vertically over the sample. 


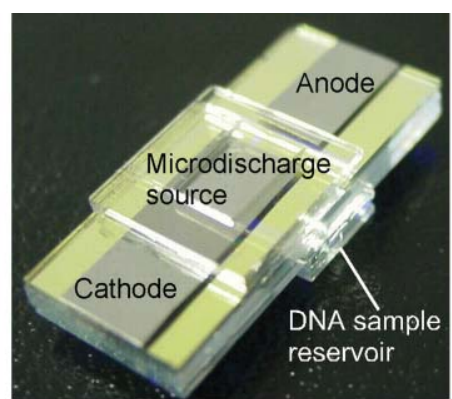

(a)

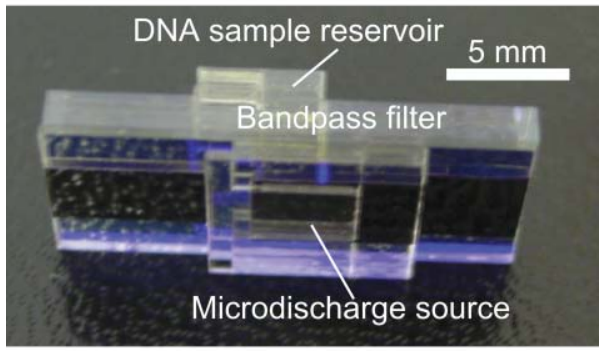

(b)

Fig. 2 Pictures of the fabricated chip. The device measures $10 \mathrm{~mm} \times$ $20 \mathrm{~mm}$.

The microdischarge is powered by a pulsed high voltage waveform generated from a rechargeable battery using the circuit shown in Fig. 3a. This particular design is based on the ringing choke converter topology, ${ }^{21}$ and consists of a transistor-transformer oscillator with secondary windings on the transformer, which can boost the voltage to up to $1000 \mathrm{~V}$.
When the switch $\mathrm{S} 1$ is closed, a small base current begins to flow into the transistor. The rising collector current leads to increasing base current due to positive feedback from the transformer. This drives the transistor into saturation. In saturation, the rate of change of current decreases, and the voltage induced in the feedback winding decreases. The base current decreases and transistor turns off. Due to the sudden decrease of collector current, a large voltage drop occurs across the primary winding, and consequently on the secondary winding. This high voltage a.c. waveform is rectified and used to charge up the discharge capacitor.

\section{Experimental results}

For validating the circuit operation, the output voltage at various test points was observed using an oscilloscope. Fig. 3b is an oscilloscope image of the output waveform, showing the repetitive nature of the voltage driving the microdischarge. The capacitor charges up to the breakdown voltage and discharges rapidly through the ionized media. As the output voltage drops the discharge vanishes. The capacitor charges up and the breakdown occurs again. This cycle repeats leading to a pulsed microdischarge. The breakdown voltage varies between 250 $280 \mathrm{~V}$. The pulse period varies between $20-30 \mathrm{~ms}$ due to the variation in breakdown voltage. The measured high voltage signal at the testpoint is shown in Fig. 3c. This signal is in accordance with the self oscillation of the circuit as described in the design section. The period of the oscillating voltage at the node marked as testpoint, is $43 \mu$ s. This signal is rectified and smoothed to charge up the discharge capacitor. The period of the pulse discharge circuit (Fig. 3b) depends mostly on breakdown voltage. The slope of the waveform charging

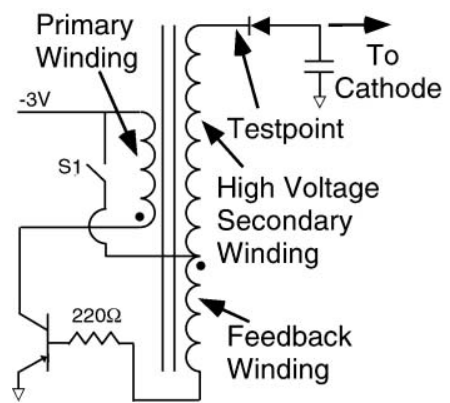

(a)

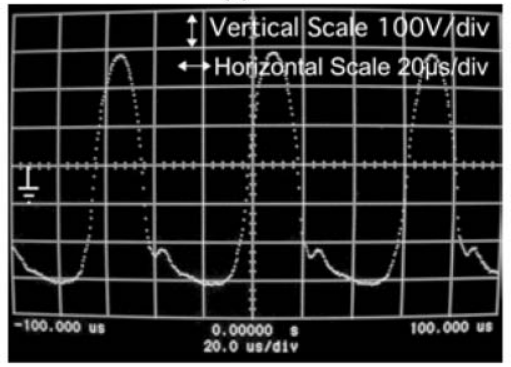

(c)

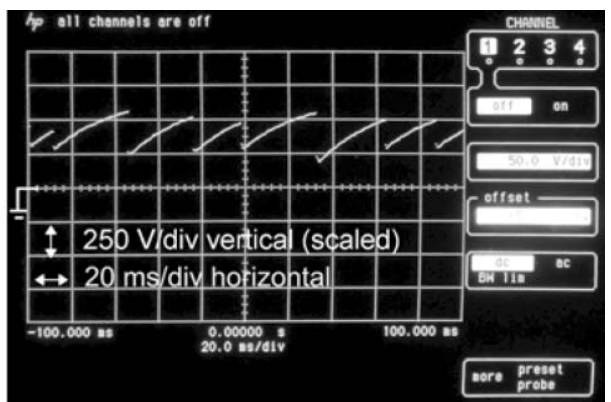

(b)

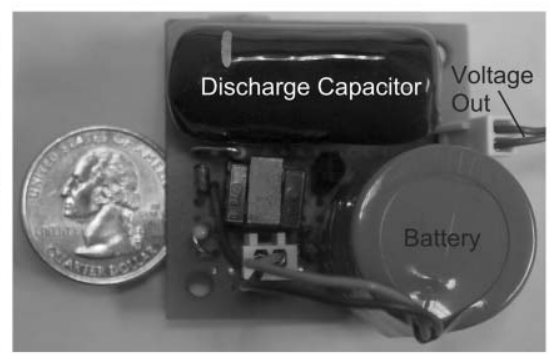

(d)

Fig. 3 Battery operated circuit used to create the discharge. (a) Schematic of the circuit. (b) Oscilloscope trace of output (cathode) waveform. The breakdown voltage varied from 240-280 V. The time period of the pulse was 20-30 ms depending on the breakdown voltage. (c) Measured waveform at the testpoint. The waveform has a frequency of $43 \mu$ s and an amplitude of $300 \mathrm{~V}$. The center line represents ground in both (b) and (c). (d) Photograph of the circuit shown against a US quarter. 
the capacitor is consistent from one cycle to the next, but the breakdown voltage varies, leading to a variation in pulse period.

For validation of the device operation, two sets of experiments were performed. In the first, a calf thymus DNA sample was labeled by SYBR green dye (Molecular Probes, Inc.), for which the absorption peaks over 491-503 $\mathrm{nm}$ and emission peaks over 510-600 nm. The liquid cathode used was $20 \% \mathrm{w} / \mathrm{v}$ $\mathrm{BaCl}_{2}$. The emission spectrum of Ba provides peaks at 454 and $493 \mathrm{~nm}$, corresponding to $25 \%$ and $97 \%$ efficiency for this dye, respectively. $^{2}$

Many intrinsic fluorophores are difficult to excite as they have low quantum efficiency, and require excitation in the deep-UV region. Tryptophan, which is a naturally occurring amino acid, is used to evaluate the device performance in the deep-UV region. It has an absorption peak over 250-290 nm and an emission peak over $280-350 \mathrm{~nm}$, with a quantum efficiency of $20 \%$. A saturated solution of lead (II) nitrate $\left(\mathrm{Pb}\left(\mathrm{NO}_{3}\right)_{2}\right)$ is used as the cathode. A UV-grade filter with a fused silica substrate (\#300-W-1D from Acton Research Corp., Acton, MA) is used between the optical source and the sample chamber. It has peak transmission wavelength of $293.0 \mathrm{~nm}$, and its full width half maximum (FWHM) is $90.1 \mathrm{~nm}$, with a peak transmittance of $47.8 \%$.

The spectra of the microdischarge for the barium chloride doped cathode are shown in Fig. 4a. The $454 \mathrm{~nm}$ and $493 \mathrm{~nm}$ lines characteristic to $\mathrm{Ba}$ are clearly seen. In the unfiltered spectrum, a number of lines characteristic to nitrogen and other atmospheric gases are also observed. The unwanted wavelengths were eliminated by the bandpass filter (350 $500 \mathrm{~nm}$ ). Fig. 4b shows the filtered spectrum. The two primary Ba lines pass through the filter, exciting the dye labeled DNA, inducing fluorescence. This fluorescence is additionally filtered by a high pass $(>500 \mathrm{~nm})$ optical filter, to reduce noise, before being imaged. The measured fluorescence image of the DNA excited by the battery-driven, on-chip source is shown in Fig. 5a. The reference image, in the absence of dye, (Fig. 5b) shows no illumination. Fig. 5c shows the image of the fluorescent DNA dye using a commercial $100 \mathrm{~W}$ mercury UV arc lamp (Osram HBO W/2) source for excitation. It is noteworthy that the measured fluorescence using the pulsed microdischarge optical source has the same quality as the commercial UV-light source.

In the second series of validation experiments L-tryptophan was used as the sample to be detected. Unlike the SYBR green tagged DNA, tryptophan has a very weak intrinsic fluorescence and requires deep UV light for excitation. This makes it particularly hard to excite, especially in microsystems. Since the working wavelengths are in UV, a spectrometer is used instead of a microscope as a detector. The filtered spectral emission obtained by a micro-discharge to the saturated $\mathrm{Pb}\left(\mathrm{NO}_{3}\right)_{2}$ solution used to excite the fluorescent sample is shown in Fig. 6a. There is a substantial peak near $280 \mathrm{~nm}$, which is characteristic of $\mathrm{Pb}$, and closely matches the excitation peak for tryptophan. Despite the optical filtering, there are a number of other peaks with high relative strength, particularly in the 300-350 $\mathrm{nm}$ emission range of tryptophan, which can interfere with the fluorescence signal. (The filter has a transmittance of $10 \%$ even at $400 \mathrm{~nm}$, which lets many of

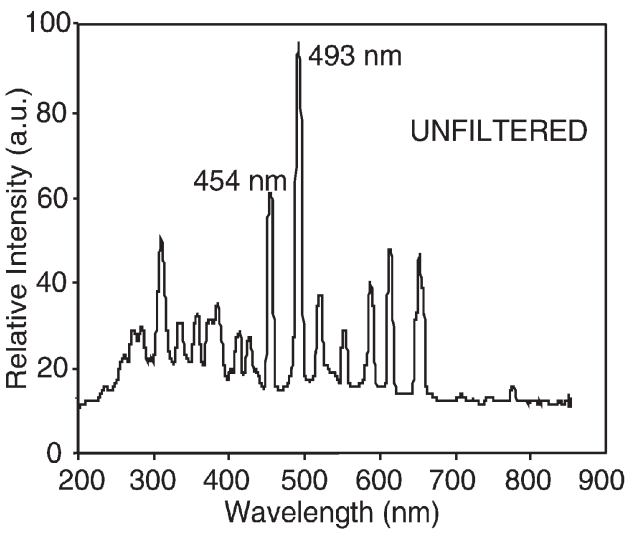

(a)

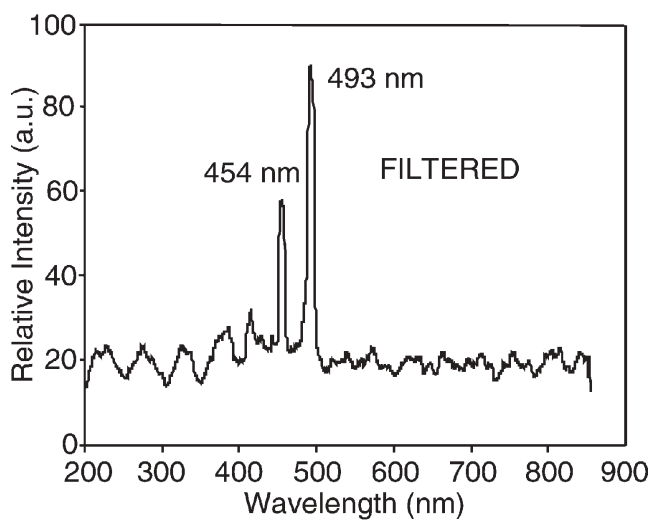

(b)

Fig. 4 Spectrum of the microdischarge source. (a) With $20 \% \mathrm{BaCl}_{2}$ solution as cathode, showing the $454 \mathrm{~nm}$ and $493 \mathrm{~nm}$ barium lines. (b) Spectrum of light after the on-chip optical filter, with all unwanted lines suppressed.

these lines through). This problem was overcome by using special techniques as outlined below.

The fluorescence sample was prepared by dissolving commercially available tryptophan in DI water at room temperature until a saturated solution was obtained. In all the measurements presented, the background radiation in the room was measured immediately before each experiment.

The absorption of the UV light was studied by placing the optical fiber on the same axis as the incident light source (i.e. along optical path I). Fig. $6 \mathrm{~b}$ shows the light spectrum after it passes through a $2.5 \mathrm{~mm}$ deep sample of tryptophan solution. We can see that tryptophan absorbs the lines in the 250 $300 \mathrm{~nm}$ range, as reported in literature. To verify this result, a control experiment was done by placing water instead of tryptophan in the sample chamber. The lines in the 250 $300 \mathrm{~nm}$ range pass through the water and can be clearly seen in the transmitted spectrum. This confirms that the tryptophan sample absorbs the light in the $250-300 \mathrm{~nm}$ range from the microdischarge source.

The fluorescence spectrum was observed by placing the fiber along optical path II, transverse to the excitation path to remove the interfering background radiation. Fig. 7a shows the observed output from a tryptophan sample, with evidence 


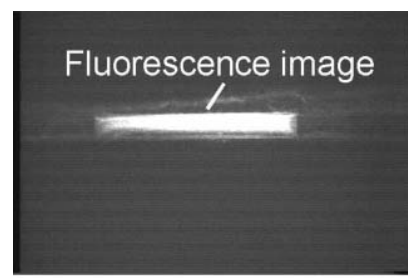

(a)

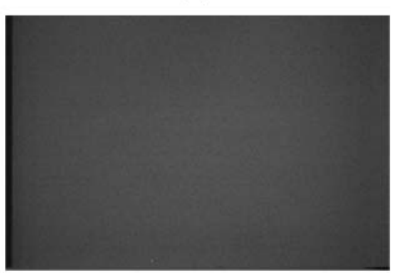

(b)

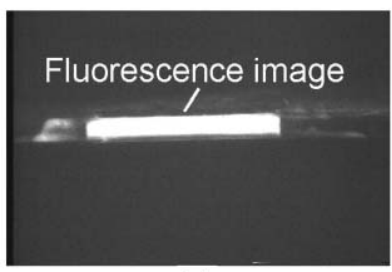

(c)

Fig. 5 (a) Photograph of the fluorescence image of SYBR green tagged DNA, using the microdischarge source as the excitation. (b) Reference image with water instead of tagged DNA, showing no fluorescence. (c) Photograph of fluorescence image of SYBR green tagged DNA using a commercial UV lamp source (100 W mercury arc lamp).

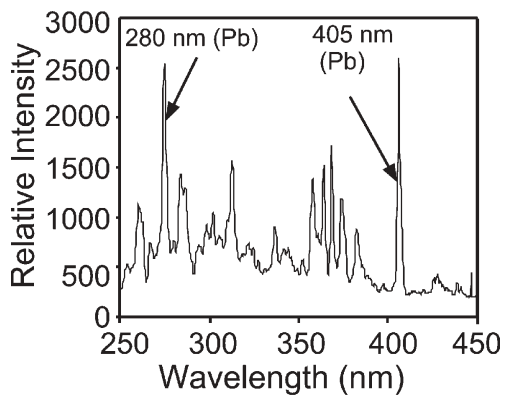

(a)

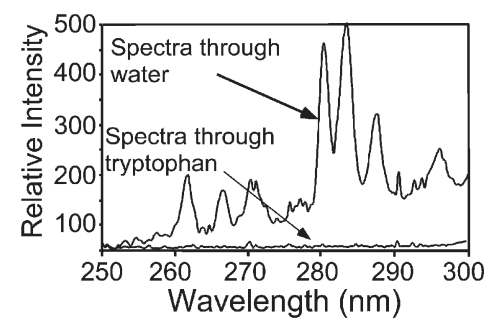

(b)

Fig. 6 (a) Filtered spectrum of the microdischarge source, with saturated $(5 \mathrm{~g}$ per $10 \mathrm{ml}) \mathrm{Pb}\left(\mathrm{NO}_{3}\right)_{2}$ solution as cathode, showing strong $280 \mathrm{~nm} \mathrm{~Pb}$ lines. This emission is used to excite the fluorescence of the tryptophan sample. (b) Light transmitted through tryptophan shows that the peaks near $280 \mathrm{~nm}$ have been absorbed. These wavelengths are transmitted through the control sample of water.

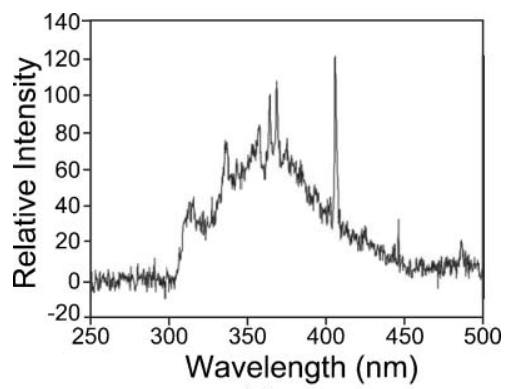

(a)

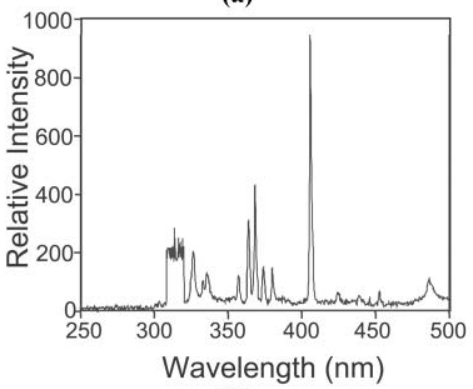

(b)

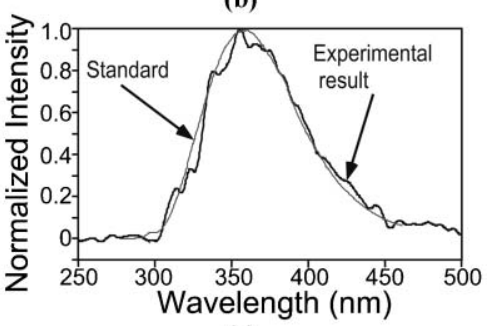

(c)

Fig. 7 (a) Optical emission measured along optical path II (as shown in Fig. 1) indicating the existence of the direct fluorescence of tryptophan by the broad peak between $325 \mathrm{~nm}$ and $425 \mathrm{~nm}$. (b) Spectrum measured with a DI water control sample in a manner analogous to Fig. 7a, shows the absence of the broad peak between $325 \mathrm{~nm}$ and $425 \mathrm{~nm}$. (c) The net output from the tryptophan sample, obtained by subtracting the curve of Fig. 7a from that of Fig. 7b scaled to the $405 \mathrm{~nm}$ peak of the spectrum in Fig. 7a. A 30-point triangular averaging was performed to reduce the noise. The reference emission curve of tryptophan from the National Bureau of Standards ${ }^{22}$ has been superimposed.

of the characteristic broad peak between $300 \mathrm{~nm}$ and $450 \mathrm{~nm}$. A control experiment with water in place of tryptophan provides the spectrum shown in Fig. $7 b$, with no evidence of the characteristic broad peak of tryptophan. The peaks near 368 and $405 \mathrm{~nm}$ are from spikes of high relative intensity in the lead nitrate spectrum which are not sufficiently attenuated by the filter and are coupled from optical path I to II. The curves in Fig. $7 \mathrm{a}$ and $7 \mathrm{~b}$ were both obtained by integrating the spectrum for $10 \mathrm{~s}$.

A simple calibration method in which the spectrum of the water sample is subtracted from that obtained with the tryptophan sample can be used to make the fluorescence emission clearer. However, before doing this, the data in Fig. 7a and 7b are scaled so that the base-to-peak amplitude at $405 \mathrm{~nm}$ is of equal magnitude in the two spectra. It is noteworthy that this calibration at $405 \mathrm{~nm}$ also removes all the other lines from the signal feed-through. Averaging over \pm 15 wavelengths using 
triangular weighted averaging is used to reduce the random noise in the spectra.

Fig. 7c shows the observed tryptophan spectrum after the signal processing, superimposed with the reference tryptophan spectrum (from ref. 22). The characteristic broad peak of tryptophan fluorescence is clear. This establishes that the microdischarge UV source can be used to excite the UV fluorescence.

\section{Conclusion}

This effort successfully demonstrated that a microfluidic chip with an integrated microdischarge source can be used to locally generate fluorescence in biochemicals. The discharges are generated in ambient air, between a metal anode and a liquid cathode, both of which are on-chip. Fluorescence of SYBR dyed calf thymus DNA is found to clearly occur, using $\mathrm{BaCl}_{2}$ solution as the cathode. As the signal quality of this fluorescence is found to be comparable to that of a commercial system, this device can be used for a variety of biomedical diagnostic applications. The characteristic fluorescence of tryptophan in a solution of water is observed using a lead nitrate solution as the source of $280 \mathrm{~nm}$ radiation used for the optical excitation of the sample. This clearly establishes the effectiveness of a microdischarge source in the deep-UV region. Since the spectral characteristics of the optical source are dependent on the spectra of metal ions in the liquid cathode, this device can be easily tuned to a variety of biological fluorescences, both extrinsic and intrinsic.

One can envision a microfluidic system in which the sample reservoir for the biochemical is part of a larger microfluidic system. Further, the reservoir for the liquid cathode can also be part of a separate fluidic system, by which the contents of the cathode can be dynamically controlled or tuned.

\section{References}

1 H. Zhu, S. M. Clark, S. C. Benson, H. S. Rye, A. N. Glazer and R. A. Mathies, High-sensitivity capillary electrophoresis of doublestranded DNA fragments using monomeric and dimeric fluorescent intercalating dyes, Anal. Chem., 1994, 66, 1941-8.

2 R. P. Haugland, Handbook of fluorescence probes and research chemicals, Molecular Probes, Eugene, OR, 1996.

3 F. W. D. Rost, Photobleaching, photoactivation, and quenching, in Quantitative Fluorescence Microscopy, Cambridge University Press, New York, 1991, pp. 115-127.

4 J. R. Lakowicz, H. Szmacinski, K. Nowaczyk and M. L. Johnson, Fluorescence lifetime imaging of free and protein-bound NADH, Proc. Natl. Acad. Sci. U. S. A., 1992, 89, 4, 1271-1275.

5 A. S. Ladokhin, Fluorescence spectroscopy in peptide and protein analysis, in Encyclopedia of Analytical Chemistry, ed. R.A. Meyers, John Wiley \& Sons Ltd., New York, 2000, pp. 5762-5779.
6 G. D. Fasman, Handbook of Biochemistry and Molecular Biology, Proteins I, CRC Press, Boca Raton, FL, 1976, pp. 183-203.

7 H. Chou, C. Spence, A. Scherer and S. Quake, A microfabricated device for sizing and sorting DNA molecules, Proc. Natl. Acad. Sci. U. S. A., 1999, 96, 11-13.

8 J. Webster, M. A. Burns, D. T. Burke and C. H. Mastrangelo, Monolithic capillary electrophoresis device with integrated fluorescence detector, Anal. Chem., 2001, 73, 1622-6.

9 M. Warren, W. C. Sweatt, J. R. Wendt, C. G. Bailey, C. M. Matzke, D. M. Arnold, S. A. Kemme, A. A. Allerman, T. R. Carter, R. E. Asbill and S. Samora, Integrated micro-optical fluorescence detection system for microfluidic electro-chromatography, Proc. SPIE-Int. Soc. Opt. Eng., 1999, 3878, 185-92.

10 E. Thrush, O. Levi, K. Wang, J. S. Harris, S. J. Smith, A. Dittmar and D. Beebe, Integrated semiconductor fluorescent detection system for biochip and biomedical applications, Proc. SPIE-Int. Soc. Opt. Eng., 2002, 4626, 289-96.

11 A. J. Fischer, A. A. Allerman, M. H. Crawford, K. H. A. Bogart, S. R. Lee, R. J. Kaplar, W. W. Chow, S. R. Kurtz, K. W. Fullmer and J. J. Figiel, Room-temperature direct current operation of $290 \mathrm{~nm}$ light-emitting diodes with milliwatt power levels, Appl. Phys. Lett., 2004, 84, 17, 3394-3396.

12 T. M. Katona, T. Margalith, C. Moe, M. C. Schimidt, S. Nakamura, J. S. Speck and S. P. DenBaars, Growth and fabrication of short wavelength UV LEDs, Proc. SPIE-Int. Soc. Opt. Eng., 2004, 5187, 250-259.

13 C. Wilson, Y. B. Gianchandani, R. R. Arslanbekov, V. Kolobov and A. E. Wendt, Profiling and modeling of DC nitrogen microplasmas, J. Appl. Phys., 2003, 94, 5, 2845-2851.

14 L. Que, C. Wilson and Y. B. Gianchandani, Microfluidic Electrodischarge devices with integrated dispersion optics for spectral analysis of water impurities, J. Microelectromech. Syst., 2005, 14, 2, 185-191.

15 G. Jenkins and A. Manz, Optical emission detection of liquid analytes using a micro-machined DC glow discharge device at atmospheric pressure, Proceedings of the Micro Total Analysis Symposium, Monterey, CA, 2001, Kluwer, Dordrecht, Netherlands.

16 C. Wilson and Y. B. Gianchandani, Spectral detection of metal contaminants in water using an on-chip microglow discharge, IEEE Trans. Electron Devices, 2002, 49, 12, 2317-2322.

17 J. Hopwood, A Microfabricated Inductively Coupled Plasma Generator, J. Microelectromech. Syst., 2000, 9, 3, 309-313.

18 L. Que, P. Selvaganapathy, B. Mitra, C. G. Wilson and Y. B. Gianchandani, Dye-Fluorescence LEd-SpEC: A Battery-Operated, On-Chip, Wavelength-Tunable Optical Source for Detection of Biochemicals, Proceedings of the Micro Total Analysis Systems Symposium, Squaw Valley, CA, October 2003, Transducers Research Foundation, Cleveland Heights, OH, pp. 1251-1254.

19 B. Mitra, C. Wilson, L. Que and Y. B. Gianchandani, A MicroFluidic Ultra-Violet Emission Source for Direct Fluorescence of Tryptophan, Proceedings of the IEEE Engineering in Medicine and Biology Society Conference, Cancun, Mexico, September 2003, IEEE Press, Piscataway, NJ, pp. 3380-3383.

20 C. Wilson, Microplasmas and microdischarges for manufacturing and sensing applications, $\mathrm{PhD}$ thesis, University of WisconsinMadison, 2003.

21 L. H. Light and P. M. Hooker, Transistor D.C. Convertors, Proc. IEE, Part B, 1955, 102, 775-786.

$22 \mathrm{R}$. Chen, Measurements of absolute values in biochemical fluorescence spectroscopy, J. Res. Natl. Bur. Stand., Sect. A, 1972, 76, 6, 593-606. 